\title{
A Fatal Case of AIDS-Defining Meningoencephalitis by C. NEOFORMANS, SEnsitive to Antifungal Therapy
}

\author{
S. Mohammad-Khani ${ }^{1}$, B. Otremba 2 , R. Klein ${ }^{3}$, H. H. Capelle ${ }^{4}$, F. Logemann ${ }^{5}$, F. C. Bange ${ }^{6}$, \\ R. E. Schmidt ${ }^{1}$, M. Stoll ${ }^{1}$
}

\footnotetext{
${ }^{1}$ Medical University of Hannover, Clinic for Immunology and Rheumatology,

${ }^{2}$ Haematooncology practice, Oldenburg,

${ }^{3}$ Medical University of Hannover, Division of Neuropathology,

${ }^{4}$ Medical University of Hannover, Clinic for Neurosurgery,

${ }^{5}$ Medical University of Hannover, Clinic for Anaesthesiology and Intensive Care,

${ }^{6}$ Medical University of Hannover, Division of Microbiology, Germany
}

\begin{abstract}
Cryptococcus neoformans is the most common cause of life threatening meningoencephalitis in HIV-infected patients. Diagnosis is based on tests for cryptoccocal antigen in serum and cerebrospinal fluid, and on culture of the organism. We present a case of AIDS-related cryptococcal meningoencephalitis unresponsive to antifungal combination therapy, despite of evidence of fungal susceptibility in vitro. Significant decreases in cryptococcal antigen titers in serum and cerebrospinal fluid did not correlate with progress in disease and fatal outcome.
\end{abstract}

Key words: Cryptococcal meningitis, HIV, antifungal therapy, cryptoccocal antigen assay

\section{INTRODUCTION}

Cryptococcosis is a life-threatening fungal infection, caused by Cryptococcus (C.) neoformans, an encapsulated yeast. Primary infection develops in the lungs by inhalation and remains asymptomatic in the majority of immunocompetent hosts [1]. T cell-related immunodeficiency, most commonly in association with HIV infection is a major predisposing factor for cerebral cryptococcosis. Disseminating disease may develop as an AIDS defining disease in HIV infected individuals predominantly in patients with $\mathrm{CD} 4+\mathrm{T}$ cell count less than $100 / \mu \mathrm{l}[2]$ - and presents in up to $80 \%$ as meningitis or meningoencephalitis [3]. Positive direct staining or culture of cerebrospinal fluid (CSF) and/or antigen testing in CSF ensures the diagnosis of cryptococcal meningitis (CM) [4] and a positive serum cryptococcal antigen (CrA) titer is suggestive for dissemination [5]. In HIV infected individuals responding to active anti retroviral therapy (HAART) and prior cryptococcal infection, cases of immune reconstitution inflammatory syndrome (IRIS) related CM have been described [6]. In a recent prospective study the incidence of CM-IRIS was found to be $17 \%$ [7]. Poor prognosis has been associated with a number of risk factors in AIDS-related CM. A serum CrA-titer $\geq 1: 512$ [8], a CSF CrA-titer $\geq$ 1:1024 [9], culture of Cryptococcus from CSF $>10^{6}$ $\mathrm{CFU} / \mathrm{ml}$, a CSF leukocyte count $\leq 20 \mathrm{cells} / \mu \mathrm{l}$, and initial alteration in mental status $[8,10-12]$. Treatment of $\mathrm{CM}$ requires induction therapy with a combination of at least two drugs. Success of antifungal induction therapy should be monitored by culturing CSF for Crytococcus. After 2 weeks of therapy with amphotericin B plus flucytosine, CSF culture results are negative in $60 \%$ to $75 \%[13,14]$.

\section{CASE REPORT}

HIV infection was diagnosed in May 2008, in a 33 year old homosexual man who presented to another hospital with fever and unproductive cough. Chest X-ray and CT-scans revealed diffuse homogeneous ground glass opacity in both lungs and pneumocystis jiroveci could be demonstrated in bronchoalveolar lavage (BAL). Standard therapy with trimethoprim-sulfamethoxazole (TMP-SMZ) and prednisolone was successful. Replicative HIV infection could be proven by PCR (91.000 copies $/ \mathrm{ml}$ ) and the CD 4 cell-count of $59 / \mu$ indicated a profound immunodeficiency. Thus HAART was started with tenofovir/emtricitabine and lopinavir/ritonavir. Two months later the patient was admitted to our clinic with increasing headache, emesis, lethargy and focal seizures for the past 4 weeks. Cerebral symptoms began with mild headaches 3 weeks prior to admission. Initially performed magnet resonance imaging (MRI) scan showed bihemispheric mass right more than left in basal ganglia area, which was not present on a MRI done 3 weeks before admission. Ring enhancement of these lesions was absent. At this point there were no signs of elevated intracranial pressure (ICP) on MRI (Fig. 1).

A lumbar punction performed on the same day revealed a CSF leukocyte count of $10 / \mu$, positive CSF CrA (1:8192) with positive India ink test. Encapsulated C. neoformans var grubii isolates growing on culture were sensitive to amphotericin B, fluconazole and flucytosine.

Serum CrA-titer was elevated with 1:32.768. Culture of CSF for bacteria, including mycobacteria remained negative, so did specific test for Treponema pallidum, Toxoplasma gondii and JC-virus. On admission the viral load was undetectable and the CD4 cell-count was still low $(44 / \mu \mathrm{l})$.

An intravenous antifungal drug combination with fluconazole $400 \mathrm{mg} / \mathrm{d}$, flucytosine $(150 \mathrm{mg} / \mathrm{kg} / \mathrm{d})$ and liposomal amphotericin B $(4 \mathrm{mg} / \mathrm{kg} / \mathrm{d})$ was administered. Due to a rapid virological response under HAART, initially an IRIS-associated CM could not be excluded. Therefore dexamethasone was given additionally to antifungal combination therapy.

Initially the patient improved mildly in mental status and serum CrA-titer dropped by 3 titer steps to 1:4096 one week after initiating antifungal therapy. 

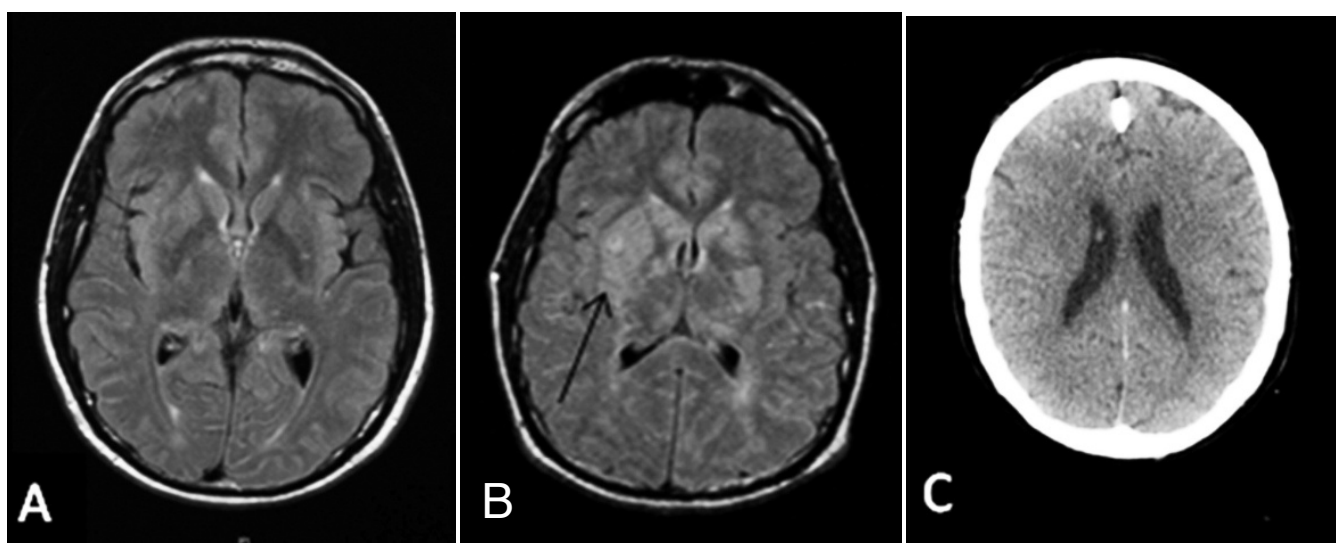

Fig. 1. Series of cerebral images in the course of infection. A) cerebral MRI 3 weeks prior to admission; B) MRI on admission with bihemispheric mass right more than left in basal ganglia area (arrow); C) Cerebral CT after 3 weeks of antifungal combination therapy with loss of gray-white distinction and effacement of cerebral sulci.

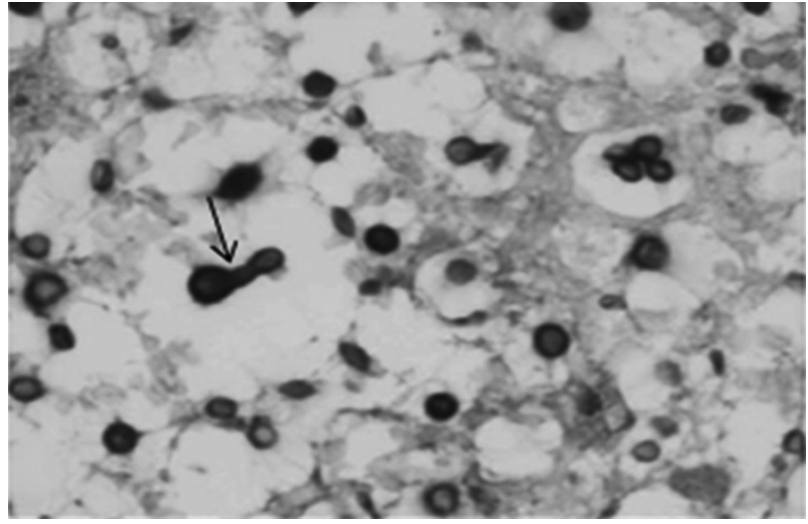

Fig. 2. Brain tissue infection with encapsulated C. neoformans appears as bubbles due to gelatinous capsules. Reproducing by budding (arrow) PAS-stain, magnification, x400.

About two weeks after starting antifungal therapy he unexpectedly clinically worsened continually with increase in seizure frequency. Another lumbar puncture performed revealed a 3 titer steps decrease of CSF CrA-titer (1:1024). Yet the cryptococci were still cutured from CSF. At this point a stereotactic brain biopsy from suspected area was done. Biopsy (Fig. 2) showed accumulation of $C$. neoformans within brain parenchyma with little cellular immune reaction and without any evidence of granulomatous encephalitis.

During biopsy, an increased opening pressure of 462 $\mathrm{mm}$ of water (physiological pressure $<200 \mathrm{~mm}$ of water) could be measured. Brain CT-scan obtained showed brain edema but no hydrocephalus, a common cause for elevated intracerebral pressure in CM (Fig. 1). Due to the risk of herniation, reduction of ICP at that time point would have require trepanation, which was denied by health care directives from the patient and his relatives.

\section{Discussion}

HIV-associated CM is an AIDS defining disease, indicating an advanced immunodeficiency.

Without therapy death by CM usually occurs within 2-4 weeks of diagnosis [12]. In a nationwide surveillance in France a significant decrease of average mortality rate in the HAART era (15.3 per 100 personyears) compared to the pre-HAART era (63.8 per 100 person-years) could be shown in AIDS defining cryptococcosis. Despite that decrease early mortality remained high [15]. In the literature CM associated mortality in patients under antifungal therapy is determined to be over $20 \%$ in the first 3 months after diagnosis $[15,16]$.

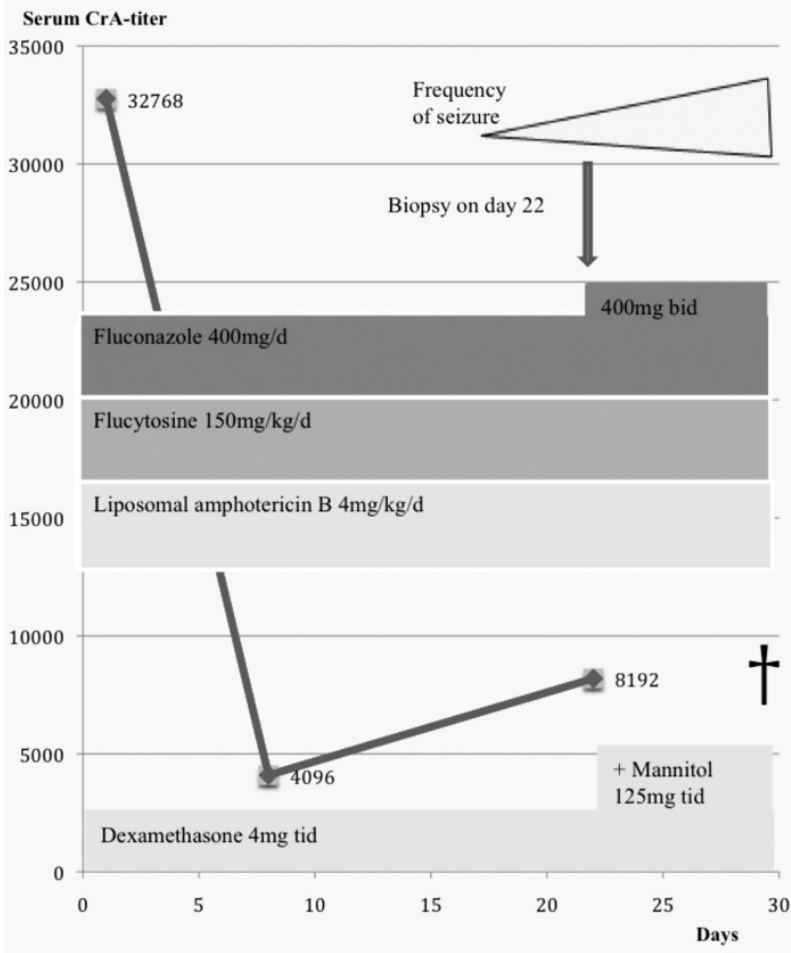

Fig. 3. Course of serum cryptococal antigen titer, antifungal antigen titer, antifungal combination therapy, ICP controlling therapy and the course of disease.

In accordance with the described risk factors the presented case fulfils criteria for severe cryptococcal cerebral infection. He initially presented with mental alteration, had a high serum CrA-titer of 1:32.768 and a CSF CrA-titer of 1:8.192, a low CSF leukocyte count of $10 / \mu \mathrm{l}$ and positive culture for C. neoformans.

A rapid establishment of antifungal activity within affected tissue should be the primary goal of any induction therapy. In Germany a triple therapy with amphotericin B, flucytosine and fluconazole is recommended for meningitis. The rate for complete remission of meningitis, which is achieved in around $80 \%$ of patients [17], may be slightly higher than under dual therapy with amphotericin B and flucytosine as favored in the United States [14].

Elevated ICP in the presented case was due to brain edema without any signs of hydrocephalus on imaging. According to the guidelines of IDSA managing of ICP by lumbar punctures or external drainage is indicated in ICP caused by hydrocephalus [18]. Due to the risk of 
herniation reduction of ICP at that time point would not allow lumbar puncture. Trepanation as the treatment of choice was denied by relatives. As an option of conservative ICP controlling therapy mannitol and sedatives were administered. However prognosis in cases with elevated ICP remains poor also in cases with internal hydrocephalus. Although invasive measures can reduce ICP, a survival benefit over 2 or 10 weeks was absent in a prospective study [16].

Clinical progress occurred despite an appropriate combination therapy with fluconazole, flucytosine and liposomal amphotericin B and evidence of fungal susceptibility - guidelines defined by CLSI 2008 [23] - in the initial as in the follow up CSF culture and indicates the limited clinical correlation of resistance tests and clinical efficacy in CM. Progress of infection is suggested by a positive CSF culture after two weeks as well by histological demonstration of C. neoformans with signs of reproducing by budding but a lack of significant inflammatory signs. Predominance of IRIS-related CM is unlikely in this case due to: (i) persistent positive culture from CSF; (ii) histological demonstration of vital C. neoformans with signs of fungal growth; (iii) absence of granulomatous inflammation, which would be the expected type of inflammation in HIV-negative patients [19] and in IRIS-related cryptoccocal infections [6].

In contrast to the clinical progression of the infection serological and CSF-titers for Cryptococcus decreased under therapy. Although sequential monitoring of elevated CrA in serum and CSF suggests to reflect directly the burden of fungal pathogen, it is potentially misleading and therefore not recommended as a surrogate marker to follow-up AIDS-related cryptococcal infection during therapy [12, 20-22].

In conclusion careful and close clinical observation, neuroimaging and direct confirmation of CSF sterility by lumbar puncture or biopsy are crucial in the monitoring during the acute phase of cryptococcal disease. As shown in the presented case a decrease of $\mathrm{CrA}$ in serum and CSF is not a reliable parameter for the response to antifungal treatment in the presented case. The primary failure of treatment occurred despite initial use of three recommended elements of an antifungal combination therapy, and in the absence of evidence for primary resistance in vitro of the isolated cryptococci.

\section{REFERENCES}

1. Mitchell TG, Perfect JR. Cryptococcosis in the era of AIDS100 years after the discovery of Cryptococcus neoformans. Clin Microbiol Rev 1995; 8:515-48.

2. French N, Gray K, Watera C, Nakiyingi J, Lugada E, Moore M, Lalloo D, Whitworth JA, Gilks CF. Cryptococcal infection in a cohort of HIV-1-infected Ugandan adults. AIDS. 2002 May 3;16(7):1031-8.

3. Manfredi R, Pieri F, Pileri SA, Chiodo F. The changing face of AIDS-related opportunism: Cryptococcosis in the highly active antiretroviral therapy (HAART) era. Case reports and literature review. Mycopathologia 1999; 148:73-8.

4. Manfredi R, Moroni A, Mazzoni A, Nanetti A, Donati M, Mastroianni A, et al. Isolated detection of cryptococcal polysaccharide antigen in cerebrospinal fluid samples from patients with AIDS. Clin Infect Dis 1996; 23:849-50.

5. Saag, MS, Graybill RA, Larsen RA, Pappas PG, Perfect JR, Powderly WG, et al. Practice guidelines for the management of cryptococcal disease. Clin Infect Dis 2000; 30:710-718.

6. Skiest DJ, Hester LJ, Hardy RD. Cryptococcal immune reconstitution inflammatory syndrome: report of four cases in three patients and review of the literature. J Infect. 2005; 5:e289-97.
7. Bicanic T, Meintjes $G$, Rebe $K$, et al. Immune reconstitution inflammatory syndrome in HIV-associated cryptococcal meningitis: a prospective study. J Acquir Immune Defic Syndr 2009; 51:130-4.

8. Dromer F, Mathoulin-Pélissier S, Launay O, Lortholary O; French Cryptococcosis Study Group.Determinants of disease presentation and outcome during cryptococcosis: the CryptoA/D study. 2007 Feb;4(2):e21.

9. Dammert P, Bustamante B, Ticona E, Llanos-Cuentas A, Huaroto L, Chávez VM, et al.Treatment of cryptococcal meningitis in Peruvian AIDS patients using amphotericin B and fluconazole. J Infect. 2008; 57:260-5.

10. Powderly WG. Current approach to the acute management of cryptococcal infections. J Infect. 2000 Jul;41(1): 18-22.

11. White M, Cirrincione C, Blevins A, Armstrong D. Cryptococcal meningitis: outcome in patients with AIDS and patients with neoplastic disease. Infect Dis 1992; 165:960-3.

12. Chuck SL, Sande MA. Infections with Cryptococcus neoformans in the acquired immunodeficiency syndrome. N Engl J Med 1989; 321:794-9.

13. Dromer F, Bernede-Bauduin C, Guillemot D, Lortholary O; French Cryptococcosis Study Group. Major role for amphotericin B-flucytosine combination in severe cryptococcosis. PLoS One. 2008 Aug 6;3(8):e2870.

14. Van der Horst CM, Saag MS, Cloud GA, Hamill RJ, Graybill JR, Sobel JD, et al. Treatment of cryptococcal meningitis associated with the acquired immunodeficiency syndrome. National Institute of Allergy and Infectious Diseases Mycoses Study Group and AIDS Clinical Trials Group. N Engl J Med 1997; 337:15-21.

15. Lortholary O, Poizat G, Zeller V, Neuville S, Boibieux A, Alvarez $\mathrm{M}$, et al. Long-term outcome of AIDS-associated cryptococcosis in the era of combination antiretroviral therapy. AIDS 2006; 20:2183-91

16. Bicanic T, Brouwer AE, Meintjes G, Rebe K, Limmathurotsakul D, Chierakul W, Teparrakkul P, Loyse A, White NJ, Wood R, Jaffar S, Harrison T. Relationship of cerebrospinal fluid pressure, fungal burden and outcome in patients with cryptococcal meningitis undergoing serial lumbar punctures. AIDS. 2009 Mar 27;23(6):701-6.

17. Weitzel A, Arasteh K, Mertenskötter T, et al. Brokmeyer NH et al.Kryptokokkosen bei HIV-positiven Patienten in Deutschland. Eine Auswertung 24 deutscher Zentren. HIVInfekt, Springer-Verlag 1999.

18. Perfect JR, Dismukes WE, Dromer F, Goldman DL, Graybill JR, Hamill RJ, Harrison TS, Larsen RA, Lortholary O, Nguyen MH, Pappas PG, Powderly WG, Singh N, Sobel JD, Sorrell TC.Clinical practice guidelines for the management of cryptococcal disease: 2010 update by the infectious diseases society of america. Clin Infect Dis. 2010 Feb 1:50(3):291-322.

19. Lee SC, Dickson DW, Casadevall A. Pathology of cryptococcal meningoencephalitis: analysis of 27 patients with pathogenic implications. Hum Pathol 1996; 27:839-47.

20. Antinori S, Radice A, Galimberti L, Magni C, Fasan M, Parravicini $\mathrm{C}$. The role of cryptococcal antigen assay in diagnosis and monitoring of cryptococcal meningitis. J Clin Microbiol 2005; 43:5828-9.

21. Aberg JA, Watson J, Segal M, Chang LW. Clinical utility of monitoring serum cryptococcal antigen (sCRAG) titers in patients with AIDS-related cryptococcal disease. HIV Clin Trials. 2000; 1:1-6.

22. Powderly WG, Cloud GA, Dismukes WE, Saag MS. Measurement of cryptococcal antigen in serum and cerebrospinal fluid: value in the management of AIDS-associated cryptococcal meningitis.Clin Infect Dis 1994; 18:789-92.

23. Clinical and Laboratory Standards Institute (CLSI) 2008. M27-A3 reference method for broth dilution antifungal susceptibility testing of yeasts; approved standard, third edition. Clinical and Laboratory Standards Institute, Wayne, PA.

Received: March 23, 2010 / Accepted: October 20, 2010

Address for correspondence:

Somayeh Mohammad-Khani

Clinic for Immunology and Rheumatology

Medical University of Hanover

Carl-Neuberg Str. 1

30625 Hanover

Germany

Tel. + 495115329672

Fax: + 49-511-5325695

E-mail: Mohammad-Khani.Somayeh@mh-hannover.de 